\title{
Intensive wave power and steel quenching 3-D model for cylindrical sample. Time direct and reverse formulations and solutions
}

\author{
Andris Buikis* and Margarita Buike \\ Institute of Mathematics and Computer Science, University of Latvia, Raina Blvd. 29, LV1459, Riga, LATVIA
}

\begin{abstract}
In this paper we develop mathematical models for three dimensional hyperbolic heat equations (wave equation or telegraph equation) with inner source power and construct their analytical solutions for the determination of the initial heat flux for cylindrical sample. As additional conditions the temperature and heat flux at the end time are given. In some cases we give expression of wave energy. In some cases we give expression of wave energy. Some solutions of time inverse problems are obtained in the form of first kind Fredholm integral equation, but others has been obtained in closed analytical form as series. We viewed both direct and inverse problems at the time. For the time inverse problem we use inversion in the time argument.
\end{abstract}

\section{INTRODUCTION}

Contrary to traditional method the intensive quenching process uses environmentally friendly highly agitated water or low concentration of water/mineral salt solutions [1]-[5]. This equation is important for wave energy generator [6, 7]. Traditionally for the mathematical description of the intensive quenching process, classical heat conduction equation is used. We have proposed to use hyperbolic heat equation [8-21] for more realistic description of the intensive quenching (IQ) process (especially for the initial stage of the process).

The idea of the usage of hyperbolic heat equation can be easily transferred to completely different sector of application - to the generation of electricity in sea or ocean by usage of wave energy [22,23]. The first known patent to use energy from ocean waves dates back to 1799 and was filed in Paris by Girard and his son [22]. It is important to note, that Ekergard and his co-authors [23] examine the development of the system in time, describing the equipment with ordinary differential equation. Here we describe the equipment in development of both - in time as well as in spatial arrangement of equipment using the three-dimensional hyperbolic heat equation. Wave power plant has to work for long time period in moving environment - waves. Therefore it is important to examine not only the development of equipment in time, but also the movement of its different components [19, 21].

Wave energy generator models can be viewed both Cartesian coordinate and cylindrical co-ordinates. In papers [11], [12], [14], [19-21] we investigate the rectangular models. Generators of cylindrical or spherical forms with fin we investigate in papers [15] and [17].
In our previous papers we have constructed various one and two dimensional analytical exact and approximate [9-16], [19-21], [24, 25] solutions for IQ processes. We consider three-dimensional statements for non-homogeneous equation with non-homogeneous boundary conditions. Such statements allow constructing mathematical models for wave power plants in connection with other equipment, for example, with wind power. In recent years, we have been able to generalize the Green's function method to areas, which consist of several canonical connected sub-areas, and thus we have obtained the exact solutions for the L-, Tand $\Pi$-type areas [9 - 11], [21].

\section{MATHEMATICAL FORMULATION OF 3-D PROBLEM FOR IQP OR WAVE POWER}

Already in the introduction we noted that Professor M. Leijon, see [23] examined the development of system in time. Here we offer to consider the description of system in time and space. For this purpose instead of the time depended ordinary differential equation, we consider the following partial differential equation:

$$
\begin{aligned}
& \frac{\partial^{2} U}{\partial t^{2}}=a_{\tau}^{2}\left[\frac{1}{r} \frac{\partial}{\partial r}\left(r \frac{\partial U}{\partial r}\right)+\frac{1}{r^{2}} \frac{\partial^{2} U}{\partial \varphi^{2}}+\frac{\partial^{2} U}{\partial z^{2}}\right]- \\
& -C U+F(r, \varphi, z, t), r \in[0, R], \varphi \in[0,2 \pi], \\
& z \in[0, l], t \in[0, T], C \geq 0, a_{\tau}^{2}=\frac{a^{2}}{\tau_{r}}, a^{2}=\frac{k}{c \rho} .
\end{aligned}
$$

\footnotetext{
* Corresponding author: buikis@1atnet.lv
} 
Here $c$ is specific heat capacity, $k$ - heat conductivity coefficient, $\rho$ - density, $\tau_{r}$ - relaxation time. The source term $\Phi(r, \varphi, z, t)$ can be from different parts of the same device or outer source, for example, wind source. In the case of wave energy we can assume different nonhomogeneous boundary conditions. Important is to formulate boundary conditions (3), (4) and (5) in the heat energy transfer form [27]:

$$
\begin{gathered}
\left.r \frac{\partial U}{\partial r}\right|_{r=0}=0, \\
\left.\left(R \frac{\partial U}{\partial r}+k_{1} U\right)\right|_{r=R}=R g_{1}(\varphi, z, t), k_{1}=\frac{R h_{3}}{k}, \\
\left.\left(\frac{\partial U}{\partial z}-k_{2} U\right)\right|_{z=0}=g_{2}(r, \varphi, t), k_{i}=\frac{h_{i}}{k}, i=2,3 \\
\left.\left(\frac{\partial U}{\partial z}+k_{3} U\right)\right|_{z=l}=g_{3}(r, \varphi, t) .
\end{gathered}
$$

Here $h_{i}$ is heat exchange coefficient. On all the other sides of device we have heat exchange with environment. In fact it is possible to look at other types of boundary conditions: first (Dirichlet) and second (Neumann) type. The initial conditions for the function $U(r, \varphi, z, t)$ are assumed in following form:

$$
\begin{aligned}
& \left.U\right|_{t=0}=U_{0}(r, \varphi, z), \\
& \left.\frac{\partial U}{\partial t}\right|_{t=0}=U_{1}(r, \varphi, z) .
\end{aligned}
$$

From the practical point of view in the steel quenching model the condition (7) can be unrealistic. The initial heat flux must be determined theoretically. As additional condition we assume that either the temperature distribution or the heat fluxes distribution at the end of process is given (known):

$\left.U\right|_{t=T}=U_{T}(r, \varphi, z)$,

$$
\left.\frac{\partial U}{\partial t}\right|_{t=T}=U_{T}^{1}(r, \varphi, z) \text {. }
$$

The formulation of the 3 dimensional mathematical model is important for wave energy generator [6], [7]. It is good see from the above point on the fig. 1 from patent [6].

For 3-D mathematical model is important that solution in $\varphi-$ direction is continuous and smooth. These 2 conditions are important for

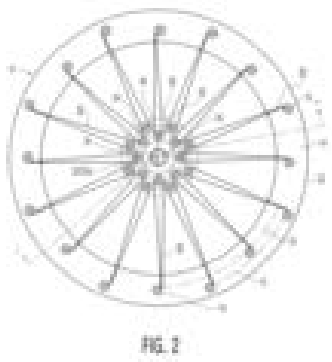

Fig. 1. The look from the above point of cylindrical piezoelectric generator from patent [6].

the reduction of 3-D model to 2-D model by conservative averaging method [9], [24] and [25]:

$$
\begin{gathered}
\left.U\right|_{\varphi=0}=\left.U\right|_{\varphi=2 \pi}, \\
\left.\frac{\partial U}{\partial \varphi}\right|_{\varphi=0}=\left.\frac{\partial U}{\partial \varphi}\right|_{\varphi=2 \pi} .
\end{gathered}
$$

\section{SOLUTION OF 3-D PROBLEM}

We assume that we have non-homogeneous KleinGordon equation-with source term: $C \geq 0$. The solution in three-dimensional problem is in following form:

$$
\begin{aligned}
& U(r, \varphi, z, t)=H(r, \varphi, z, t)+\int_{0}^{R} \xi d \xi \int_{0}^{2 \pi} d \varsigma \times \\
& \int_{0}^{l} U_{1}(\xi, \eta, \varsigma) G(r, \varphi, z, \xi, \eta, \varsigma, t) d \eta+\int_{0}^{2 \pi} d \varsigma \times \\
& \int_{0}^{R} \xi d \xi \int_{0}^{l} U_{0}(\xi, \eta, \varsigma) \frac{\partial}{\partial t} G(r, \varphi, z, \xi, \eta, \varsigma, t) d \eta .
\end{aligned}
$$

Here

$$
\begin{aligned}
& H(r, \varphi, z, t)=a_{\tau}^{2} R^{2} \int_{0}^{t} d \tau \int_{0}^{2 \pi} d \varsigma \times \\
& \int_{0}^{l} g_{1}(\eta, \varsigma, \tau) G(r, \varphi, z, R, \eta, \varsigma, t-\tau) d \eta-a_{\tau}^{2} \int_{0}^{t} d \tau \times \\
& \int_{0}^{2 \pi} d \varsigma \int_{0}^{R} \xi g_{2}(\xi, \varsigma, \tau) G(r, \varphi, z, \xi, \varsigma, 0, t-\tau) d \xi+a_{\tau}^{2} \times \\
& \int_{0}^{t} d \tau \int_{0}^{2 \pi} d \varsigma \int_{0}^{R} \xi g_{3}(\xi, \varsigma, \tau) G(r, \varphi, z, \xi, \varsigma, l, t-\tau) d \xi+ \\
& \int_{0}^{t} d \tau \int_{0}^{2 \pi} d \varsigma \int_{0}^{R} \xi d \xi \times
\end{aligned}
$$


$\int_{0}^{l} F(\xi, \eta, \varsigma, \tau) G(r, \varphi, z, \xi, \eta, \varsigma, t-\tau) d \eta$

The Green function [26], [27] for initial-boundary problem for Klein-Gordon equation is known; see [28]:

$$
\begin{aligned}
& G(r, \varphi, z, \xi, \eta, \zeta, t)=\frac{1}{\pi} \sum_{n=0}^{\infty} \sum_{m=1}^{\infty} A_{n} \times \\
& \sum_{s=1}^{\infty} \frac{\mu_{n m}^{2} J_{n}\left(\mu_{n m} r\right) J_{n}\left(\mu_{n m} \xi\right)}{\left(\mu_{n m}^{2} R^{2}+k_{1}^{2} R^{2}-n^{2}\right)\left[J_{n}\left(\mu_{n m} R\right)\right]^{2}} \times \\
& \frac{\cos [n(\varphi-\eta)] h_{s}(z) h_{s}(\zeta) \sin \left(\lambda_{n m s} t\right)}{\left\|h_{s}\right\|^{2} \lambda_{n m s}} .
\end{aligned}
$$

Here $J_{n}(\xi)-$ is Bessel's function and

$$
\begin{aligned}
& \lambda_{n m s}=\sqrt{a_{\tau}^{2}\left(\mu_{n m}^{2}+\beta_{s}^{2}\right)+C}, \\
& A_{n}=\left\{\begin{array}{l}
1, \text { if } n=0, \sqrt{ } \\
2, \text { if } n>0 ;
\end{array}\right. \\
& h_{s}(z)=\cos \left(\beta_{s} z\right)+\frac{k_{2}}{\beta_{s}} \sin \left(\beta_{s} z\right), \\
& \left\|h_{s}\right\|^{2}=\frac{k_{3}\left(\beta_{s}^{2}+k_{2}^{2}\right)}{2 \beta_{s}^{2}\left(\beta_{s}^{2}+k_{3}^{2}\right)}+\frac{k_{2}}{2 \beta_{s}^{2}}+\frac{l}{2}\left(1+\frac{k_{2}^{2}}{\beta_{s}^{2}}\right) .
\end{aligned}
$$

The eigenvalues $\mu_{n m}, \beta_{s}$ are positive roots of the transcendental equations:

$$
\mu J_{n}^{\prime}(\mu R)+k_{1} J_{n}(\mu R)=0, \frac{\operatorname{tg}(\beta l)}{\beta}=\frac{k_{2}+k_{3}}{\beta^{2}-k_{2} k_{3}} .
$$

We assume that at final moment $t=T$ is known only one boundary condition (8). Then from solution (12) we easy obtain Fredholm first type integral equation with respect to function $U_{1}(r, \varphi, z)$ :

$$
\begin{aligned}
& \int_{0}^{R} \xi d \xi \int_{0}^{2 \pi} d \varsigma \int_{0}^{l} U_{1}(\xi, \eta, \varsigma) G(r, \varphi, z, \xi, \eta, \varsigma, T) d \eta \\
& =\Phi(r, \varphi, z) .
\end{aligned}
$$

The unknown right side function $\Phi(r, \varphi, z)$ is in the following form:

$\Phi(r, \varphi, z)=U_{T}(r, \varphi, z)-H(r, \varphi, z, T)-$
$\left.\int_{0}^{2 \pi} d \varsigma \int_{0}^{R} \xi d \xi \int_{0}^{l} U_{0}(\xi, \eta, \varsigma) \frac{\partial}{\partial t} G(r, \varphi, z, \xi, \eta, \varsigma, t)\right|_{t=T} d \eta$.

Similar situation is, if is done second boundary condition (9). We differentiate solution (12) regarding time:

$$
\frac{\partial}{\partial t} U(r, \varphi, z, t)=\frac{\partial}{\partial t} H(r, \varphi, z, t)+
$$

$$
\begin{aligned}
& \int_{0}^{R} \xi d \xi \int_{0}^{2 \pi} d \varsigma \int_{0}^{l} U_{1}(\xi, \eta, \varsigma) \frac{\partial}{\partial t} G(r, \varphi, z, \xi, \eta, \varsigma, t) d \eta+ \\
& \int_{0}^{2 \pi} d \varsigma \int_{0}^{R} \xi d \xi \int_{0}^{l} U_{0}(\xi, \eta, \varsigma) \frac{\partial^{2}}{\partial t^{2}} G(r, \varphi, z, \xi, \eta, \varsigma, t) d \eta .
\end{aligned}
$$

We obtain again 1st kind Fredholm integral equation for the determination of unknown initial heat flux:

$$
\begin{aligned}
& \left.\int_{0}^{R} \xi d \xi \int_{0}^{2 \pi} d \varsigma \int_{0}^{l} U_{1}(\xi, \eta, \varsigma) \frac{\partial}{\partial t} G(r, \varphi, z, \xi, \eta, \varsigma, t)\right|_{t=T} d \eta \\
& =\Phi_{1}(r, \varphi, z) .
\end{aligned}
$$

Here

$$
\begin{aligned}
& \Phi_{1}(r, \varphi, z)=U_{T}^{1}(r, \varphi, z)-\left.\frac{\partial}{\partial t} H(r, \varphi, z, t)\right|_{t=T}- \\
& \left.\int_{0}^{2 \pi} d \varsigma \int_{0}^{R} \xi d \xi \int_{0}^{l} U_{0}(\xi, \eta, \varsigma) \frac{\partial^{2}}{\partial t^{2}} G(r, \varphi, z, \xi, \eta, \varsigma, t)\right|_{t=T} d \eta .
\end{aligned}
$$

There is an interesting situation, if both additional conditions (8), (9) are known. In this case we introduce new time argument by formula

$$
\tilde{t}=T-t \text {. }
$$

The formulation for new function $V(r, \varphi, z, \tilde{t})_{\text {with }}$ time variable $\tilde{t}$ is following:

$\frac{\partial^{2} V}{\partial \tilde{t}^{2}}=a_{\tau}^{2}\left[\frac{1}{r} \frac{\partial}{\partial r}\left(r \frac{\partial V}{\partial r}\right)+\frac{1}{r^{2}} \frac{\partial^{2} V}{\partial \varphi^{2}}+\frac{\partial^{2} V}{\partial z^{2}}\right]-$

$-C V+F(r, \varphi, z, T-\tilde{t})$,

$\left.\left(R \frac{\partial V}{\partial r}+k_{1} V\right)\right|_{r=R}=R g_{1}(\varphi, z, T-\tilde{t})$,

$$
\begin{aligned}
& \left.\left(\frac{\partial V}{\partial z}-k_{2} V\right)\right|_{z=l}=g_{2}(r, \varphi, T-\tilde{t}), \\
& \left.\left(\frac{\partial V}{\partial z}+k_{3} V\right)\right|_{z=0}=g_{3}(r, \varphi, T-\tilde{t}), \\
& \left.V\right|_{\tilde{t}=0}=U_{T}(r, \varphi, z),\left.\frac{\partial V}{\partial \tilde{t}}\right|_{\tilde{t}=0}=-U_{T}^{1}(r, \varphi, z) .
\end{aligned}
$$

Similar to (10) the solution of inverse problem looks like:

$$
\begin{aligned}
& V(x, y, z, \tilde{t})=H(x, y, z, \tilde{t})-\int_{0}^{R} \xi d \xi \int_{0}^{2 \pi} d \varsigma \times \\
& \int_{0}^{l} U_{T}^{1}(\xi, \eta, \varsigma) G(r, \varphi, z, \xi, \eta, \varsigma, \tilde{t}) d \eta+\int_{0}^{2 \pi} d \varsigma \times
\end{aligned}
$$

$\int_{0}^{R} \xi d \xi \int_{0}^{l} U_{T}(\xi, \eta, \varsigma) \frac{\partial}{\partial \tilde{t}} G(r, \varphi, z, \xi, \eta, \varsigma, \tilde{t}) d \eta$.

There is no problem to transform the expression for $H(x, y, z, \tilde{t})$ in following form: 


$$
\begin{aligned}
& H(x, y, z, \tilde{t})=a_{\tau}^{2} R \int_{T-\tilde{t}}^{T} d \tau \int_{0}^{2 \pi} d \varsigma \times \\
& \int_{0}^{l} g_{1}(\eta, \varsigma, \tau) G(r, \varphi, z, R, \eta, \varsigma, T-\tau) d \eta-a_{\tau}^{2} \int_{T-\tilde{t}}^{T} d \tau \\
& \int_{0}^{2 \pi} d \varsigma \int_{0}^{R} g_{2}(\xi, \varsigma, \tau) G(x, y, z, \xi, 0, \varsigma, T-\tau) d \xi+a_{\tau}^{2} \times \\
& \int_{T-\tilde{t}}^{T} d \tau \int_{0}^{2 \pi} d \varsigma \int_{0}^{R} g_{3}(\xi, \varsigma, \tau) G(x, y, z, \xi, b, \varsigma, T-\tau) d \xi+ \\
& \int_{T-\tilde{t}}^{T} d \tau \int_{0}^{2 \pi} d \varsigma \times \\
& \int_{0}^{R} d \xi \int_{0}^{l} F(\xi, \eta, \varsigma, \tau) G(r, \varphi, z, \xi, \eta, \varsigma, T-\tau) d \eta .
\end{aligned}
$$

For the heat flux in time from (19) we have the expression:

$$
\begin{aligned}
& \frac{\partial}{\partial \tilde{t}} V(r, \varphi, z, \tilde{t})=\frac{\partial}{\partial \tilde{t}} H(r, \varphi, z, \tilde{t})+ \\
& \int_{0}^{R} \xi d \xi \int_{0}^{2 \pi} d \varsigma \int_{0}^{l} V_{1}(\xi, \eta, \varsigma) \frac{\partial}{\partial \tilde{t}} G(r, \varphi, z, \xi, \eta, \varsigma, \tilde{t}) d \eta+ \\
& \int_{0}^{2 \pi} d \varsigma \int_{0}^{R} \xi d \xi \int_{0}^{l} V_{0}(\xi, \eta, \varsigma) \frac{\partial^{2}}{\partial \tilde{t}^{2}} G(r, \varphi, z, \xi, \eta, \varsigma, \tilde{t}) d \eta .
\end{aligned}
$$$$
\text { From last expression at } \tilde{t}=T \text { and equality (17) we }
$$$$
\text { have solution for the time inverse problem: }
$$$$
U_{T}^{1}(r, \varphi, z)=-\left.\frac{\partial}{\partial \tilde{t}} H(r, \varphi, z, \tilde{t})\right|_{\tilde{t}=T}-
$$$$
\left.\int_{0}^{R} \xi d \xi \int_{0}^{2 \pi} d \varsigma \int_{0}^{l} V_{1}(\xi, \eta, \varsigma) \frac{\partial}{\partial \tilde{t}} G(r, \varphi, z, \xi, \eta, \varsigma, \tilde{t})\right|_{\tilde{t}=T} d \eta-
$$$$
\left.\int_{0}^{2 \pi} d \varsigma \int_{0}^{R} \xi d \xi \int_{0}^{l} V_{0}(\xi, \eta, \varsigma) \frac{\partial^{2}}{\partial \tilde{t}^{2}} G(r, \varphi, z, \xi, \eta, \varsigma, \tilde{t})\right|_{\tilde{t}=T} d \eta
$$

Very interesting is wave energy [29] as you can see in [21]:

$$
I_{0}(t)=\sum_{n=0}^{\infty} \sum_{m=1}^{\infty} \sum_{s=1}^{\infty} \frac{\sin ^{2}\left(\lambda_{n m s} t\right)}{\lambda_{n m s}^{2}} .
$$

\section{SOLUTION OF 3-D PROBLEM WITH CONSTANT INITIAL CONDITIONS}

In the previous section we have constructed some three dimensional solutions for direct and time inverse problems for hyperbolic heat equation. Enough often initial conditions are constant functions [21], [25]. In this case it is to solve the solutions in the form of series. For simplicity we look the homogeneous boundary conditions:

$$
\begin{aligned}
& U(r, \varphi, z, t)=U_{1} \int_{0}^{R} \xi d \xi \int_{0}^{2 \pi} d \varsigma \times \\
& \int_{0}^{l} G(r, \varphi, z, \xi, \eta, \varsigma, t) d \eta+U_{0} \int_{0}^{2 \pi} d \varsigma \times \\
& \int_{0}^{R} \xi d \xi \int_{0}^{l} \frac{\partial}{\partial t} G(r, \varphi, z, \xi, \eta, \varsigma, t) d \eta= \\
& =U_{0} G_{0}+U_{1} G_{1} .
\end{aligned}
$$

We use the Green function form (14) in the little different form:

$$
\begin{aligned}
& G(r, \varphi, z, \xi, \eta, \zeta, t)=\frac{1}{\pi} \times \\
& \sum_{n=0}^{\infty} \sum_{m=1}^{\infty} \sum_{s=1}^{\infty} \frac{A_{n} \mu_{n m}^{2} J_{n}\left(\mu_{n m} r\right) J_{n}\left(\mu_{n m} \xi\right)}{\left(\mu_{n m}^{2} R^{2}+k_{1}^{2} R^{2}-n^{2}\right)\left[J_{n}\left(\mu_{n m} R\right)\right]^{2}} \times
\end{aligned}
$$$$
\frac{[\cos (n \varphi) \cos (n \eta)+\sin (n \varphi) \sin (n \eta)]}{\left\|h_{s}\right\|^{2}} \times
$$

$$
\frac{h_{s}(z) h_{s}(\zeta) \sin \left(\lambda_{n m s} t\right)}{\lambda_{n m s}} \text {. }
$$

The function $G_{0}$ after integration can be obtained in following form:

$$
\begin{aligned}
& G_{0}=\frac{1}{\pi} \sum_{n=0}^{\infty} \sum_{m=1}^{\infty} \sum_{s=1}^{\infty} \frac{A_{n} \mu_{n m}^{2} J_{n}\left(\mu_{n m} r\right) \cos \left(\lambda_{n m s} t\right)}{\left(\mu_{n m}^{2} R^{2}+k_{1}^{2} R^{2}-n^{2}\right)\left[J_{n}\left(\mu_{n m} R\right)\right]^{2}} \times \\
& \frac{[\cos (n \varphi) \sin (n l)+\sin (n \varphi)(1-\cos (n \eta))]}{n\left\|h_{s}\right\|^{2}} h_{s}(z) \times \\
& \frac{\sin \left(2 \pi \beta_{s}\right)+\frac{k_{2}}{\beta_{s}}\left(1-\cos \left(2 \pi \beta_{s}\right)\right)_{R}}{\beta_{s}} \xi J_{n}\left(\mu_{n m} \xi\right) d \xi .
\end{aligned}
$$

Similarly we can transform the function $G_{1}$ :

$$
\begin{aligned}
& G_{1}=\frac{1}{\pi} \sum_{n=0}^{\infty} \sum_{m=1}^{\infty} \sum_{s=1}^{\infty} \frac{A_{n} \mu_{n m}^{2} J_{n}\left(\mu_{n m} r\right) \sin \left(\lambda_{n m s} t\right)}{\left(\mu_{n m}^{2} R^{2}+k_{1}^{2} R^{2}-n^{2}\right)\left[J_{n}\left(\mu_{n m} R\right)\right]^{2}} \times \\
& \frac{[\cos (n \varphi) \sin (n l)+\sin (n \varphi)(1-\cos (n \eta))]}{n\left\|h_{s}\right\|^{2}} h_{s}(z) \times \\
& \frac{\sin \left(2 \pi \beta_{s}\right)+\frac{k_{2}}{\beta_{s}}\left(1-\cos \left(2 \pi \beta_{s}\right)\right)_{R}}{\beta_{s}} \int_{0} \xi J_{n}\left(\mu_{n m} \xi\right) d \xi .
\end{aligned}
$$

In this paper we can shown that time reverse problem with two final time conditions is not ill-posed problem and can be solved similarly as time direct problem. It was shown in our paper [21] that for rectangular sample time reverse problem can be solved without some numerical problem. It is good known that for inverse problem is not easy to calculate the solution [30] - [34]. 


\section{CONCLUSIONS}

We have constructed some three dimensional solutions for direct and time inverse problems for hyperbolic heat equation. The solutions for determination of initial heat flux are obtained either in the form of Fredholm integral equation of 1 st kind with continuous kernel or in the closed analytical form - in the form of series. In the future we will try to solve two dimensional and one dimensional problem for hyperbolic heat equation. These solutions can be obtained from of three dimensional solution by conservative averaging method. For the intensive steel quenching method with constant initial conditions we obtain the solution in the form of series. As second step we can use this method for different geometries.

This work has been supported by Latvian Council of Sciences (grant 623/2014).

\section{References}

1. N.I. Kobasko. Steel Quenching in Liquid Media under Pressure. - Kyiv, Naukova Dumka (1980).

2. N. I. Kobasko. Intensive Steel Quenching Methods, Handbook "Theory and Technology of Quenching”, Springer-Verlag (1992).

3. M.A. Aronov, N. Kobasko, J.A. Powell. Intensive Quenching of Carburized Steel Parts, IASME Transactions, Issue 9, 2, p. 1841-1845 (2005).

4. N.I. Kobasko. Transient Nucleate Boiling Process to Be Widely for Super Strengthening of Materials and Obtaining Other Benefits in Heat Treating Industry. UA Patent No. 109935, Published on Oct. 26, Bulletin No. 20, (2015).

5. N.I. Kobasko. Real and effective heat transfer coefficients (HTCs) used for computer simulation of transient nuclear boiling processes during quenching. Materials Performance and Characteristics, 1, No. 1, p.1-20, (2012).

6. S.H. Salter. Apparatus for use in the extraction of energy from waves on water. US Patient 4,134,023. January 14, (1977).

7. C.B. Carroll. Piezoelectric rotary electrical energy generator. US Patient 6194815 B1. February 27, (2001).

8. M. Buike, A. Buikis. Approximate Solutions of Heat Conduction Problems in Multi- Dimensional Cylinder Type Domain by Conservative Averaging Method, Part 1. Proceedings of the $5^{\text {th }}$ IASME/WSEAS Int. Conf. on Heat Transfer, Thermal Engineering and Environment, Vouliagmeni, Athens, August 25 -27, p. $15-20,(2007)$.
9. M. Buike, A. Buikis. Hyperbolic heat equation as mathematical model for steel quenching of L-shape samples, Part 1 (Direct Problem). Applied and Computational Mathematics. Proceedings of the 13th WSEAS International Conference on Applied Mathematics (MATH'08), Puerto De La Cruz, Tenerife, Canary Islands, Spain, December 15-17, WSEAS Press, p. 198-203, (2008).

10. T. Bobinska, M. Buike, A. Buikis. Hyperbolic Heat Equation as Mathematical Model for Steel Quenching of L-Shape Samples, Part 2 (Inverse Problem). Proceedings of 5th IASME/WSEAS International Conference on Continuum Mechanics (CM'10), University of Cambridge, UK, February 23-25, p. 2126, (2010).

11. M. Buike, A. Buikis. Several Intensive Steel Quenching Models for Rectangular Samples. Proceedings of NAUN/WSEAS International Conference on Fluid Mechanics and Heat \&Mass Transfer, Corfu Island, Greece, July 22-24. p. 88-93 (2010)

12. T. Bobinska, M. Buike, A. Buikis. Hyperbolic Heat Equation as Mathematical Model for Steel Quenching of L-and T-Shape Samples, Direct and Inverse Problems. Transactions of Heat and Mass Transfer. Vol.5, Issue 3, July 2010. p. 63-72.

13. S. Blomkalna, A. Buikis. Heat conduction problem for double-layered ball. Progress in Industrial Mathematics at ECMI 2012. Springer, p. 417-426, (2014).

14. T. Bobinska, M. Buike, A. Buikis. Comparing solutions of hyperbolic and parabolic heat conduction equations for L-shape samples. Recent Advances in Fluid Mechanics and Heat @Mass Transfer. Proceedings of the $9^{\text {th }}$ IASME/WSEAS International Conference on THE'11. Florence, Italy, August 2325, p. 384-389, (2011).

15. A. Piliksere, A. Buikis. Analytical solution for intensive quenching of cylindrical sample. Proceedings of 6th International Scientific Colloquium "Modelling for Material Processing", Riga, September 16-17, p. 181-186, (2010).

16. A. Piliksere, M. Buike, A. Buikis. Steel quenching process as hyperbolic heat equation for cylinder. Proceedings of $6^{\text {th }}$ Baltic Heat Conference BHTC2011, ISBN 978-952-15-2640-4 (CD-ROM), (2011).

17. S. Blomkalna, M. Buike, A. Buikis. Several intensive steel quenching models for rectangular and spherical samples. Recent Advances in Fluid Mechanics and Heat \& Mass Transfer. Proceedings of the $9^{\text {th }}$ 
IASME/WSEAS International Conference on THE'11. Florence, Italy, August 23-25, p. 390-395, (2011).

18. A. Buikis, H. Kalis. Hyperbolic type approximation for the solutions of the hyperbolic heat conduction equation in 3-D domain. Mathematical and Computational Methods in Applied Sciences. Proceedings of the 3rd International Conference on Applied, Numerical and Computational Mathematics (ICANCM'15). Sliema, Malta, August 17-19, pp. 4251, (2015).

19. M. Buike, A. Buikis, H. Kalis. Wave energy and steel quenching models, which are solved exactly and approximately. Mathematical and Computational Methods in Applied Sciences. Proceedings of the 3rd International Conference on Applied, Numerical and Computational Mathematics (ICANCM'15). Sliema, Malta, August 17-19, pp. 72-81, (2015).

20. A. Buikis, H. Kalis. Hyperbolic Heat Equation in Bar and Finite Difference Schemes of Exact Spectrum. Latest Trends on Theoretical and Applied Mechanics, Fluid Mechanics and Heat \& Mass Transfer. WSEAS Press, pp. 142-147, (2010).

21. M. Buike, A. Buikis, H. Kalis. Time Direct and Time Inverse Problems for Wave Energy and Steel Quenching Models, Solved Exactly and Approximately. WSEAS Transactions on Heat and Mass Transfer. Vol. 10, p. 30-43, (2015).

22.Wikipedia: http://en.wikipedia.org/wiki/Wave power.

23. B. Ekergard, V. Castellucci, A. Savin, M. Leijon. Axial Force Damper in a Linear Wave Energy Convertor. Development and Applications of Oceanic Engineering. Vol. 2, Issue 2, May, (2013).

24. A. Buikis. Conservative averaging as an approximate method for solution of some direct and inverse heat transfer problems. Advanced Computational Methods in Heat Transfer, IX. WIT Press, p. 311-320, (2006).

25. R. Vilums, A. Buikis. Conservative averaging method for partial differential equations with discontinuous coefficients. WSEAS Transactions on Heat and Mass Transfer. 1, Issue 4, p. 383-390, (2006).

26. G.F. Roach. Green's Functions. Cambridge University Press, (1999).
27. H.S. Carslaw, C.J. Jaeger. Conduction of Heat in Solids. Oxford, Clarendon Press, (1959).

28. A.D. Polyanin. Handbook of Linear Partial Differential Equations for Engineers and Scientists. Chapman \& Hall/CRC, (Russian edition, 2001). (2002).

29. L. Debnath. Nonlinear Partial Differential Equations for Scientists and Engineers. 2nd ed. Birkhäuser, (2005).

30. M. Buike, A. Buikis, R. Vilums. One-Dimensional Intensive Steel Quenching Models. Recent Advances in Mechanical Engineering. Proceedings of the $5^{\text {th }}$ International Conference on Fluid Mechanics and Heat \& Mass Transfer. Lisbon, Portugal, October 30November 1, p. 54-62, (2014).

31. A. Buikis, S. Guseinov. Some one-dimensional coefficients inverse model problems of the heat transfer. - Proceedings of the Latvian Academy of Sciences, Sec. B, 57, No 3/4 (626), pp.133-137, (2003).

32. A. Buikis, Sh. Guseinov. Solution of Reverse Hyperbolic Heat equation for intensive carburized steel quenching. Proceedings of ICCES'05 (Advances in Computational and Experimental Engineering and Sciences). December 1-6, IIT Madras. p. 741-746, (2005)

33. A. Buikis, Sh. Guseinov, M. Buike. Modelling of Intensive Steel Quenching Process by Time Inverse Hyperbolic Heat Conduction. Proceedings of the $4^{\text {th }}$ International Scientific Colloquium "Modelling for Material Processing”. Riga, June 8-9, p. 169-172, (2006).

34. A. Buikis, Sh. Guseinov. Conservative averaging method for solutions of inverse problems of mathematical physics. Progress in Industrial Mathematics at ECMI 2002. A. Buikis, R. Ciegis, A. D. Fitt (Eds.), Springer, p. 241-246, (2004). 